\title{
Exhaled carbon monoxide in asthmatics: a meta-analysis
}

\author{
Jingying Zhang ${ }^{1}$, Xin Yao*1, Rongbin Yu², Jianling Bai², Yun Sun ${ }^{1}$, Mao Huang*1, lan M Adcock ${ }^{3}$ and Peter J Barnes ${ }^{3}$
}

\begin{abstract}
Background: The non-invasive assessment of airway inflammation is potentially advantageous in asthma management. Exhaled carbon monoxide (eCO) measurement is cheap and has been proposed to reflect airway inflammation and oxidative stress but current data are conflicting. The purpose of this meta-analysis is to determine whether eCO is elevated in asthmatics, is regulated by steroid treatment and reflects disease severity and control.

Methods: A systematic search for English language articles published between 1997 and 2009 was performed using Medline, Embase and Cochrane databases. Observational studies comparing eCO in non-smoking asthmatics and healthy subjects or asthmatics before and after steroid treatment were included. Data were independently extracted by two investigators and analyzed to generate weighted mean differences using either a fixed or random effects metaanalysis depending upon the degree of heterogeneity.

Results: 18 studies were included in the meta-analysis. The eCO level was significantly higher in asthmatics as compared to healthy subjects and in intermittent asthma as compared to persistent asthma. However, eCO could not distinguish between steroid-treated asthmatics and steroid-free patients nor separate controlled and partly-controlled asthma from uncontrolled asthma in cross-sectional studies. In contrast, eCO was significantly reduced following a course of corticosteroid treatment.

Conclusions: $\mathrm{eCO}$ is elevated in asthmatics but levels only partially reflect disease severity and control. eCO might be a potentially useful non-invasive biomarker of airway inflammation and oxidative stress in nonsmoking asthmatics.
\end{abstract}

\section{Background}

Asthma is a chronic inflammatory disorder of the airways, associated with airway hyperresponsiveness (AHR) that leads to recurrent episodes of wheezing, breathlessness, chest tightness, and coughing, particular at night or in the early morning [1]. The assessment of airway inflammation is likely to play an important role in the future management of asthma. However, the "gold standard" for assessment of airway inflammation, bronchial biopsy, is invasive and too expensive to perform on a regular basis. Hence, much attention is paid to the non-invasive measurement of airway inflammation, such as induced sputum and of surrogates such as exhaled nitric oxide (eNO).

\footnotetext{
* Correspondence: yaoxin@njmu.edu.cn, hm6114@126.com Department of Respiratory Disease, The First Affiliated Hospital of Nanjing Medical University, 300 Guangzhou Road, Nanjing, China Full list of author information is available at the end of the article
}

The level of eNO is elevated in asthmatics, even when asymptomatic [2-4], and shows a good response to corticosteroids [5,6]. However, the measurement of eNO is expensive, limiting its use in primary care and most specialist clinics.

Some studies have reported that exhaled carbon monoxide (eCO), which is used as an indicator of smoking [7], is significantly increased in asthmatics not treated with corticosteroids $[8,9]$. CO in the body is mainly derived from the degradation of hemoglobin by the enzyme heme oxygenase (HO) [10]. It has been found that the expression of the inducible isoform, $\mathrm{HO}-1$, is increased in alveolar macrophages in asthmatics in association with increased exhaled CO [9]. These findings suggested that eCO may be a candidate of non-invasive biomarker of airway inflammation.

However, several studies have failed to replicate these early results $[11,12]$. We therefore performed a metaanalysis of the published evidence to determine whether $\mathrm{eCO}$ is increased in asthmatic adults and children, and 
whether eCO may reflect the clinical severity or be influenced by corticosteroids.

\section{Methods}

A comprehensive literature search from 1997 to 2009 in English language was performed in Medline (using PubMed as the search engine), Embase and Cochrane databases. The search terms were 'exhaled CO', 'expired CO', 'carbon monoxide', 'asthma', 'bronchial spasm', 'bronchoconstriction', 'bronchial hyperreactivity', 'airway inflammation', 'wheeze' and 'wheezing'. (Additional file 1)

A priori inclusion criteria was: 1 ) observational studies; 2) comparing eCO levels between adults or children with an established diagnosis of asthma and healthy control subjects or comparing eCO levels in the same asthmatic population before and after steroid treatment; 3) both asthmatic patients and healthy subjects were nonsmokers, since smoking status could significantly affect $\mathrm{eCO}$ levels [7]. Exclusion criteria included: 1) asthmatic patients with systemic diseases, such as diabetes, sepsis $[13,14]$ and 2) studies without healthy subjects as the control group.

A systematic search including a title screen, abstract review, and full text article review. Each study was evaluated independently by two investigators (JZ and YS). Any disagreements about adjudications were resolved through the third party (XY). For each eligible study, we extracted data including number of participants (n), age, gender, clinical feature, treatment, method measuring $\mathrm{eCO}$, and the mean value (mean), standard deviation (SD), 95\% confidence interval (CI), or standard error of mean (SEM) of exhaled CO. SEM or 95\%CI was transformed to SD, using standard statistical conversions.

Review Manager (Version 5, The Cochrane Collaboration) was used to generate the weighted mean differences (WMD) of eCO between asthmatics and healthy subjects. $I^{2}$ statistic was tested for heterogeneity. We used a fixed effects model when $I^{2}$ was equal or less than $50 \%$, and a random effects model when $I^{2}$ was greater than $50 \%$. Meta-regression analysis on the time of publication was performed by STATA (Version 9.2, STATA Corporation, College Station). Subgroup analysis was performed, according to: 1) whether the study group consisted of adult or children, 2) whether the participants were treated with corticosteroids (systemic or inhaled), 3) the clinical severity of asthma. Publication bias was evaluated through visual inspection of funnel plots, the Begg's test [15] and the Egger's Asymmetry test [16], calculating by STATA. Publication bias may be present if $P$ value is less than 0.05 .

\section{Results}

Figure 1 shows the results of the search for articles. Of the total 644 articles, 184 duplicates and 349 irrelevant stud- ies were excluded after screening the titles. A further 82 articles failed to meet the inclusion criteria after abstract review. Among the remaining 29 articles, 6 studies did not have healthy control groups and 5 studies did not provide sufficient eCO data such as mean value, $\mathrm{SD}$, $95 \% \mathrm{CI}$ or SEM. We subsequently tried to contact the authors by email to obtain further information without success. Finally, 18 articles were included in our metaanalysis [17-34]. 4 studies directly provided SD of eCO, 2 studies used $95 \% \mathrm{CI}$ and the remaining studies provided SEM. As no unequal variances were mentioned in these studies, the classical t-test was used to transform $95 \% \mathrm{CI}$ into SD. The characteristics of the 18 studies were listed in the Additional file 2, 3. There were no evidence of publication bias (Begg's test: $\mathrm{P}=0.843$; Egger's Asymmetry test: $\mathrm{P}=0.211$; Figure 2 shows the Funnel plot). The heterogeneity between different studies is significant $\left(I^{2}=\right.$ $75 \%$ ), therefore, we performed a meta-regression analysis on the time of publication. The $\tau^{2}$ estimate is 0.3782 , which means the time of publication could only partly explain the heterogeneity.

\section{Overall eCO levels in asthmatics and healthy subjects}

Data was pooled from 15 studies irrespective of the age of the participants, the severity of disease or treatment. The overall WMD of eCO between asthmatics and healthy subjects was $1.25 \mathrm{ppm}$ (95\% CI 0.92 to $1.58 ; I^{2}=75 \%$; Random effects model). Asthmatic patients showed significantly higher eCO levels as compared to healthy subjects. (Figure 3)

\section{2. eCO levels in childhood and adult asthma}

There were 7 articles focused on childhood asthma and 7 on adult asthma. The remaining article [31] studied a population above 10 years old and, therefore, could not be included as either childhood or adult asthma. The WMD of eCO between asthmatic children and healthy children was $0.95 \mathrm{ppm}$ (95\% CI 0.65 to $1.24 ; I^{2}=59 \%$; Random effects model). And the WMD of eCO between asthmatic adults and healthy adults was $1.49 \mathrm{ppm}(95 \%$ CI 0.84 to $2.14 ; I^{2}=73 \%$; Random effects model). Both asthmatic children and asthmatic adults showed significant higher eCO levels compared to healthy children and healthy adults respectively. (Figure 4)

\section{3. eCO levels in steroid-free and steroid-treated asthmatics}

13 articles mentioned steroid-free asthmatics (defined as either steroid naïve or not receiving regular inhaled or systemic corticosteroids and treated by on-demand $\beta_{2}-$ agonist), and 8 articles mentioned steroid-treated asthmatics (defined as current use of inhaled or systemic corticosteroids irrespective of $\beta_{2}$-agonist or montelukast use). The WMD of eCO was $1.39 \mathrm{ppm}(95 \% \mathrm{CI} 0.82$ to $1.95 ; I^{2}=91 \%$; Random effects model) between steroid- 


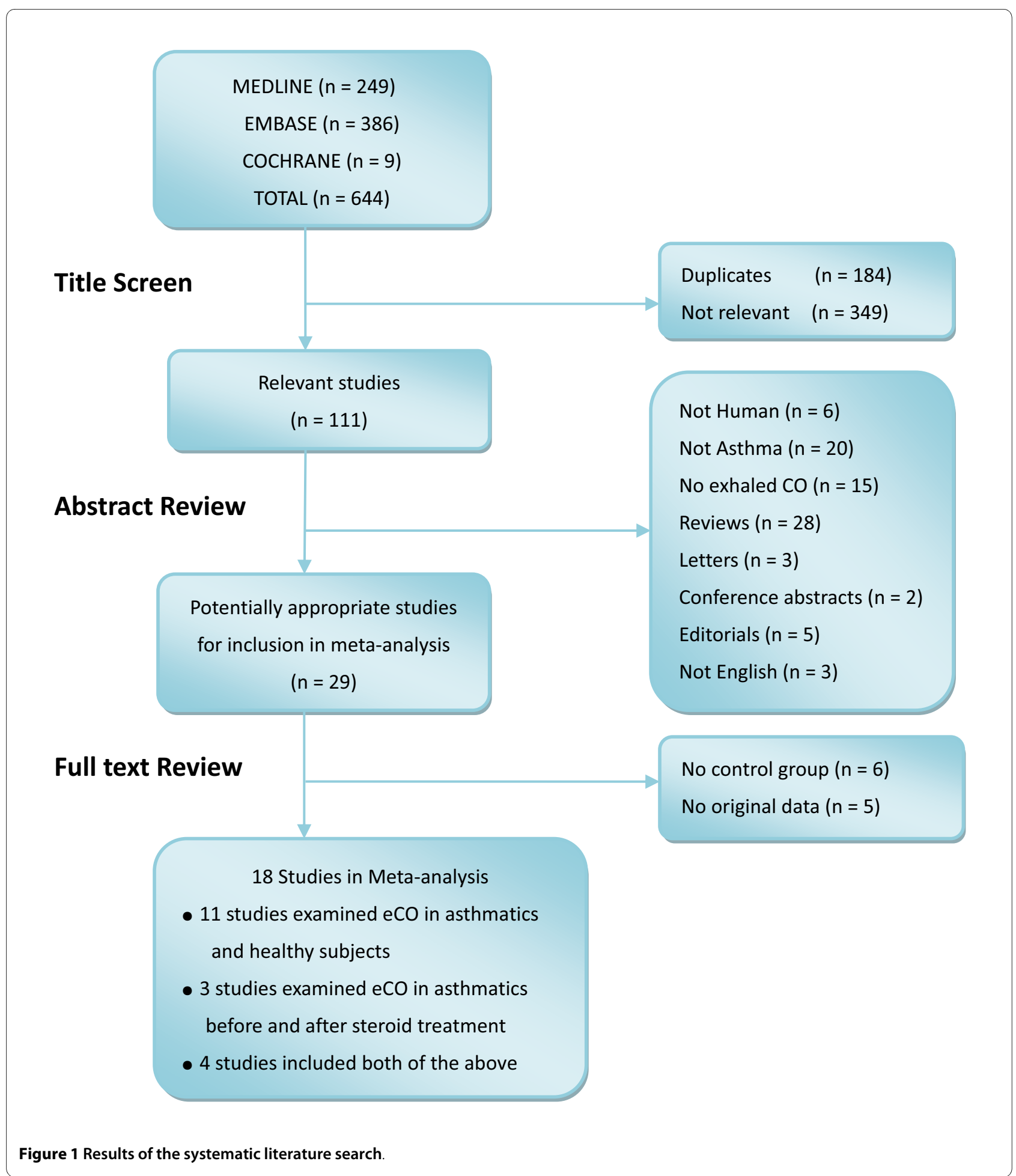

free asthmatics and healthy subjects, and $0.79 \mathrm{ppm}$ ( $95 \%$ CI 0.35 to $1.23 ; I^{2}=84 \%$; Random effects model) between steroid-treated asthmatics and healthy subjects. Both steroid-free and steroid-treated asthmatics had significantly higher eCO levels than healthy subjects. Steroid-treated asthmatic patients had lower eCO levels compared to steroid-free asthmatic patients $(0.79 \mathrm{ppm}$ vs
$1.39 \mathrm{ppm}$ ) but this failed to reach statistical significance. (Figure 5)

\section{4. eCO levels in different asthma severities}

According to the GINA [1] criteria, there are 4 categories of disease severity: intermittent, mild persistent, moderate persistent and severe persistent asthma. However, due 


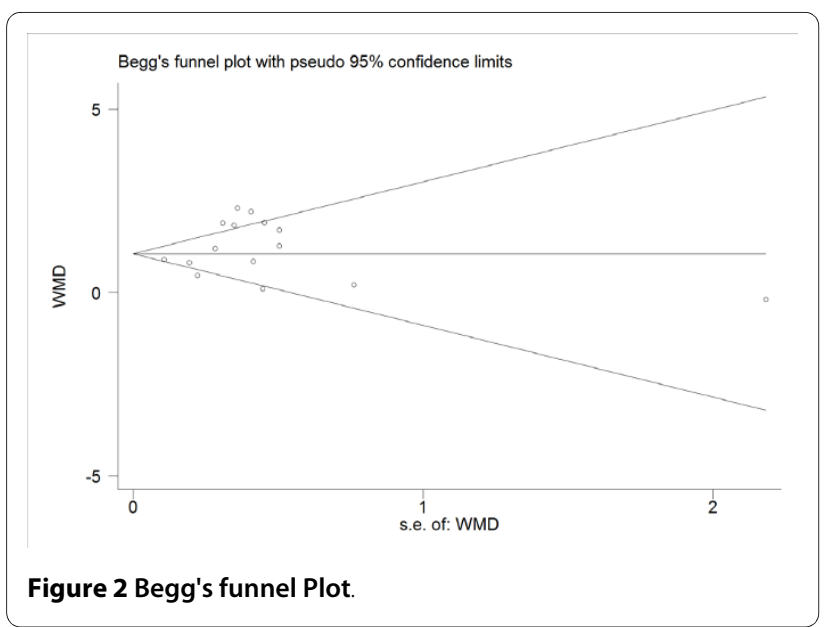

to the limited information provided by the articles, we could not differentiate mild persistent asthma from moderate persistent asthma. Therefore, we separated the subjects into three groups: intermittent asthma (4 articles), mild + moderate persistent asthma (8 articles) and severe persistent asthma ( 2 articles). The WMD of eCO was 0.31 ppm ( $95 \%$ CI 0.16 to $0.46 ; I^{2}=11 \%$; Fixed effects model) between intermittent asthmatics and healthy subjects (Figure 6), $0.84 \mathrm{ppm}\left(95 \% \mathrm{CI} 0.48\right.$ to $1.20 ; I^{2}=62 \%$; Random effects model) between mild + moderate persistent asthmatics and healthy subjects, and $2.20 \mathrm{ppm}$ (95\% CI 0.29 to $4.10 ; I^{2}=68 \%$; Random effects model) between severe persistent asthmatics and healthy subjects (Figure 7). The eCO levels were higher across all asthma severities as compared to healthy subjects. Furthermore, both mild + moderate persistent and severe persistent asthmatics showed significantly higher eCO levels than intermittent asthmatics. However, the eCO level of mild + moderate persistent asthma did not differ significantly from that of severe persistent asthma.

\section{5. eCO levels in different asthma control states}

We attempted to classify asthma control status into 3 categories: controlled, partly controlled and uncontrolled [1]. However, some studies did not provide enough information to distinguish between controlled and partly controlled disease. Thus, we divided subjects into 2 groups: controlled + partly controlled group ( 6 articles) and uncontrolled group ( 3 articles). The WMD of eCO was $1.20 \mathrm{ppm}\left(95 \% \mathrm{CI} 0.39\right.$ to $2.01 ; I^{2}=91 \%$; Random effects model) between controlled + partly controlled asthmatics and healthy subjects, and $2.12 \mathrm{ppm}$ (95\%CI 0.56 to $3.68 ; I^{2}$ = 95\%; Random effects model) between uncontrolled asthmatics and healthy subjects. Both controlled + partly controlled asthma and uncontrolled asthma showed significantly higher eCO levels as compared to healthy subjects. There was no statistical difference in eCO levels between controlled + partly controlled and uncontrolled asthma. (Figure 8)

\section{6. eCO levels in asthmatics before and after steroid treatment}

There were 7 articles focusing on the influence of corticosteroid treatment on $\mathrm{eCO}$, including 4 studies with inhaled corticosteroids, 2 studies with oral glucocorticoids and the remaining study with both inhaled and oral corticosteroids (the details of the studies are shown in

\begin{tabular}{|c|c|c|c|c|c|c|c|c|c|c|}
\hline \multirow{2}{*}{ Study or Subgroup } & \multicolumn{3}{|c|}{ Asthmatic Subjects } & \multicolumn{3}{|c|}{ Healthy Subjects } & \multirow[b]{2}{*}{ Weight } & \multirow{2}{*}{$\begin{array}{l}\text { Mean Difference } \\
\text { IV, Random, } 95 \% \mathrm{Cl}\end{array}$} & \multirow{2}{*}{\multicolumn{2}{|c|}{$\begin{array}{l}\text { Mean Difference } \\
\text { IV, Random. } 95 \% \mathrm{Cl}\end{array}$}} \\
\hline & Mean & SD & Total & Mean & SD & Total & & & & \\
\hline Antczak 2005 & 3.1 & 2.04 & 8 & 3.3 & 7.98 & 15 & $0.6 \%$ & $-0.20[-4.48,4.08]$ & & \\
\hline Rosias 2004 & 2.3 & 1.44 & 23 & 2.1 & 2.1 & 9 & $3.4 \%$ & $0.20[-1.29,1.69]$ & & \\
\hline Yamaya 2001 & 2.85 & 3.98 & 71 & 1.15 & 0.8 & 20 & $5.5 \%$ & $1.70[0.71,2.69]$ & & $\longrightarrow$ \\
\hline Beck-Ripp 2004 & 4.99 & 1.74 & 15 & 3.72 & 1.03 & 20 & $5.5 \%$ & $1.27[0.28,2.26]$ & & $\longrightarrow$ \\
\hline Grover 2008 & 5.76 & 2.24 & 42 & 3.86 & 1.35 & 21 & $6.0 \%$ & $1.90[1.01,2.79]$ & & $\longrightarrow$ \\
\hline Khatri 2001 & 1.9 & 1.13 & 8 & 1.8 & 0.49 & 6 & $6.1 \%$ & $0.10[-0.78,0.98]$ & & - \\
\hline Montuschi 1999 & 3.74 & 1.9 & 44 & 2.9 & 0.95 & 10 & $6.5 \%$ & $0.84[0.03,1.65]$ & & \\
\hline Zayasu 1997 & 3.7 & 3.06 & 60 & 1.5 & 0.55 & 30 & $6.6 \%$ & $2.20[1.40,3.00]$ & & $\longrightarrow$ \\
\hline Paredi 1999 & 4.4 & 1.27 & 18 & 2.1 & 1.22 & 37 & $7.2 \%$ & $2.30[1.59,3.01]$ & & $\longrightarrow$ \\
\hline Ohara 2006 & 2.83 & 2.38 & 51 & 1 & 1.37 & 188 & $7.4 \%$ & $1.83[1.15,2.51]$ & & $\longrightarrow$ \\
\hline Harvath 1998 & 4.79 & 1.93 & 62 & 2.9 & 1.15 & 37 & $7.9 \%$ & $1.89[1.28,2.50]$ & & $\longrightarrow$ \\
\hline Zanconate 2002 & 3.2 & 1.1 & 30 & 2 & 0.92 & 21 & $8.3 \%$ & $1.20[0.64,1.76]$ & & 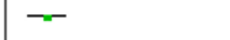 \\
\hline Ece 2000 & 1.32 & 1.5 & 54 & 0.86 & 1.35 & 235 & $9.1 \%$ & $0.46[0.02,0.90]$ & & - \\
\hline Uasuf 1999 & 1.82 & 0.82 & 29 & 1.01 & 0.76 & 40 & $9.5 \%$ & $0.81[0.43,1.19]$ & & - \\
\hline Yilmaz 2003 & 1.83 & 0.85 & 117 & 0.94 & 0.5 & 45 & $10.4 \%$ & $0.89[0.68,1.10]$ & & $=$ \\
\hline Total $(95 \% \mathrm{Cl})$ & & & 632 & & & 734 & $100.0 \%$ & $1.25[0.92,1.58]$ & & $\bullet$ \\
\hline \multicolumn{9}{|c|}{$\begin{array}{l}\text { Heterogeneity: } \text { Tau }^{2}=0.26 ; \mathrm{Chi}^{2}=55.11, \mathrm{df}=14(\mathrm{P}<0.00001) ; \mathrm{I}^{2}=75 \% \\
\text { Test for overall effect: } Z=7.45(\mathrm{P}<0.00001)\end{array}$} & $\begin{array}{cc}-4 & -2 \\
\text { Healthy Subjects }\end{array}$ & $\begin{array}{lcc}0 & 2 & 4 \\
\text { Asthmatic Subjects }\end{array}$ \\
\hline
\end{tabular}

Figure 3 Overall eCO levels in asthmatics. The WMD of eCO levels between asthmatics and healthy subjects is 1.25 ppm (95\%Cl: 0.92 to 1.58$)$ using the random effects model. Test for heterogeneity $R=75 \%$. SD: standard deviation; IV: inverse variance; Cl: confidence interval; WMD: weighted mean difference; eCO: exhaled carbon monoxide. 


\begin{tabular}{|c|c|c|c|c|c|c|c|c|c|c|}
\hline \multirow[b]{2}{*}{ Stucty or Subqroup } & \multicolumn{3}{|c|}{ Asthmatic Subjects } & \multicolumn{3}{|c|}{ Healthy Subjects } & \multirow{2}{*}{\multicolumn{2}{|c|}{$\begin{array}{c}\text { Mean Difference } \\
\text { Weight } N, \text { Random, 95\% } \mathrm{Cl}\end{array}$}} & \multirow{2}{*}{\multicolumn{2}{|c|}{$\begin{array}{l}\text { Mean Difference } \\
\text { N, Random, 95\% } \mathrm{Cl}\end{array}$}} \\
\hline & & SD & Total & Mean & SD & Total & & & & \\
\hline \multicolumn{11}{|l|}{ 2.1.1 Children } \\
\hline Rosias 2004 & 2.3 & 1.44 & 23 & 2.1 & 2.1 & 9 & $3.5 \%$ & $0.20[-1.29,1.69]$ & & - \\
\hline Beck-Ripp 2004 & 4.99 & 1.74 & 15 & 3.72 & 1.03 & 20 & $6.9 \%$ & $1.27[0.28,2.26]$ & & 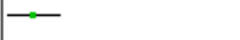 \\
\hline Ohara 2006 & 2.83 & 2.38 & 51 & 1 & 1.37 & 188 & $11.4 \%$ & $1.83[1.15,2.51]$ & & - \\
\hline Zanconate 2002 & 3.2 & 1.1 & 30 & 2 & 0.92 & 21 & $14.3 \%$ & $1.20[0.64,1.76]$ & & - \\
\hline Ece 2000 & 1.32 & 1.3 & 54 & 0.86 & 1.35 & 235 & $19.3 \%$ & $0.46[0.07,0.85]$ & & $=$ \\
\hline Uasuf 1999 & 1.82 & 0.82 & 29 & 1.01 & 0.76 & 40 & $19.5 \%$ & $0.81[0.43,1.19]$ & & $=$ \\
\hline Yilmaz 2003 & 1.83 & 0.85 & 117 & 0.94 & 0.5 & 45 & $25.1 \%$ & $0.89[0.68,1.10]$ & & 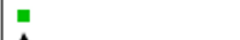 \\
\hline Subtotal $(95 \% \mathrm{Cl})$ & & & 319 & & & 558 & $100.0 \%$ & $0.95[0.65,1.24]$ & & 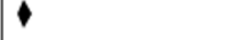 \\
\hline \multicolumn{11}{|c|}{$\begin{array}{l}\text { Heterogeneity: } \text { Tau }^{2}=0.08 ; \mathrm{Chi}^{2}=14.77, \mathrm{df}=6(P=0.02) ; \mathrm{I}^{2}=59 \% \\
\text { Test for overall effect: } Z=6.23(P<0.00001)\end{array}$} \\
\hline \multicolumn{11}{|l|}{ 2.1.2 Adults } \\
\hline Antczak 2005 & 3.1 & 2.04 & 8 & 3.3 & 7.98 & 15 & $2.1 \%$ & $-0.20[-4.48,4.08]$ & & \\
\hline Yamaya 2001 & 2.85 & 3.98 & 71 & 1.15 & 0.8 & 20 & $14.4 \%$ & $1.70[0.71,2.69]$ & & -- \\
\hline Khatri 2001 & 1.9 & 1.13 & 8 & 1.8 & 0.49 & 6 & $15.5 \%$ & $0.10[-0.78,0.98]$ & & - \\
\hline Montuschi 1999 & 3.74 & 1.9 & 44 & 2.9 & 0.95 & 10 & $16.2 \%$ & $0.84[0.03,1.65]$ & & - \\
\hline Zayasu 1997 & 3.7 & 3.06 & 60 & 1.5 & 0.55 & 30 & $16.3 \%$ & $2.20[1.40,3.00]$ & & $\rightarrow$ \\
\hline Paredi 1999 & 4.4 & 1.27 & 18 & 2.1 & 1.22 & 37 & $17.3 \%$ & $2.30[1.59,3.01]$ & & $=$ \\
\hline Howath1998 & 4.79 & 1.93 & 62 & 2.9 & 1.15 & 37 & $18.2 \%$ & $1.89[1.28,2.50]$ & & 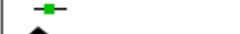 \\
\hline Subtotal $(95 \% \mathrm{Cl})$ & & & 271 & & & 155 & $100.0 \%$ & $1.49[0.84,2.14]$ & & 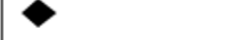 \\
\hline \multicolumn{11}{|c|}{$\begin{array}{l}\text { Heterogeneity: } \mathrm{Tau}^{2}=0.51 ; \mathrm{Chi}^{2}=22.16, \mathrm{df}=6(\mathrm{P}=0.001) ; \mathrm{I}^{2}=73 \% \\
\text { Test for overall effect: } Z=4.51(\mathrm{P}<0.00001)\end{array}$} \\
\hline & & & & & & & & & $\begin{array}{ccc}-4 & -2 & 0 \\
\text { Healthy Subjects }\end{array}$ & Asthmatic Subjects \\
\hline \multicolumn{11}{|c|}{$\begin{array}{l}\text { Figure } 4 \text { eCO levels in childhood and adult asthma. The WMD of eCO levels between asthmatic children and healthy children is } 0.95 \mathrm{ppm}(95 \% \mathrm{Cl} \text { : } \\
0.65 \text { to 1.24) using the random effects model. Test for heterogeneity } R=59 \% \text {. The WMD of eCO levels between asthmatic adults and healthy adults } \\
\text { is } 1.49 \mathrm{ppm}(95 \% \mathrm{Cl}: 0.84 \text { to } 2.14) \text { using random effects model. Test for heterogeneity } R=73 \% \text {. SD: standard deviation; IV: inverse variance; Cl: confi- } \\
\text { dence interval; WMD: weighted mean difference; eCO: exhaled carbon monoxide. }\end{array}$} \\
\hline
\end{tabular}

Additional file 3). The WMD of eCO in asthmatics before and after steroid treatment was $1.98 \mathrm{ppm}(95 \% \mathrm{CI} 0.53$ to 3.43; $I^{2}=94 \%$; Random effects model) indicating that eCO was significantly reduced by steroid treatment. (Figure 9)

\section{Discussion}

This meta-analysis based on 15 studies in 632 asthmatics and 734 healthy subjects demonstrates that $\mathrm{eCO}$ was increased in both adults and children with asthma, irrespective of steroid treatment, disease severity or level of asthma control (Figure 10). Apart from asthma, other diseases such as allergic rhinitis, bronchiectasis, lower and upper respiratory tract infections, interstitial lung disease and cystic fibrosis have also shown an increased production of eCO [10]. eCO was even found to be elevated in asymptomatic atopic subjects [35]. These findings suggest that increased eCO level may be an indicator of airway inflammation but it does not discriminate between diseases.

Endogenous $\mathrm{CO}$ in the expired air is mainly derived from the degradation of hemoglobin by $\mathrm{HO}$ [10]. Many mediators present in inflamed airways, including interleukin- $1 \beta$, tumor necrosis factor- $\alpha$, interferon- $\gamma$ and hydrogen peroxide could induce $\mathrm{HO}-1$ [35]. In vivo chal- lenge with allergen, but not methacholine, also results in increased eCO levels, suggesting that an inflammatory response and not bronchoconstriction per se is necessary to enhance HO-1 activity and/or expression [36]. HO- 1 is present in alveolar macrophages; however, there is conflicting evidence as to whether $\mathrm{HO}-1$ is expressed in the airway epithelium and whether HO-1 is up-regulated in asthmatics $[9,37,38]$. Harju et al [38] showed that HO-1 was up-regulated in the alveolar macrophages of steroidfree asthmatics, and Horvath et al [9] also found increased expression of HO-1 in alveolar macrophages in asthmatics associated with increased exhaled CO. In contrast, Lim et al [37] demonstrated that HO-1 was equally expressed in alveolar macrophages from asthmatics and healthy subjects. This discrepancy was explained by the possibility that the airway was already exposed to daily oxidant stresses such as environment pollutants, and therefore the expression of HO-1 was probably already maximal in asthmatics and healthy subjects. Alternatively, the techniques used may not have been sensitive enough to quantify differences when $\mathrm{HO}-1$ was highly expressed and enzymic activity was not measured.

The level of eCO in intermittent asthmatics was significantly lower than mild + moderate persistent and severe persistent asthmatics, suggesting that eCO may be corre- 


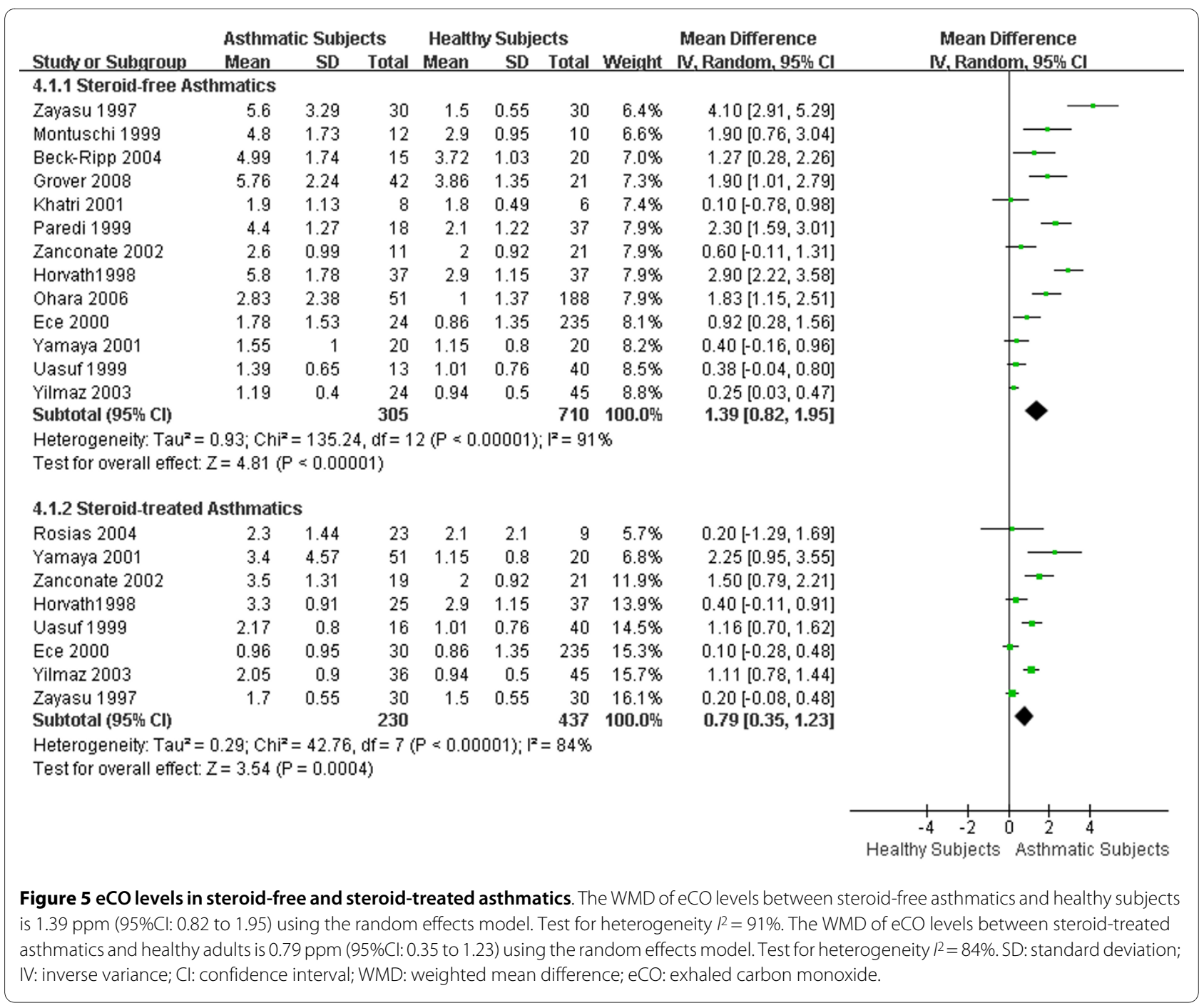

lated with disease severity. However, no statistical significance was found between mild + moderate persistent asthmatics and severe persistent asthmatics. There was much greater variability in the $95 \% \mathrm{CI}$ of WMD for eCO in severe persistent asthma compared with other groups. This probably reflects the fact that only 2 studies were included in this group and the sample size (46 severe persistent asthmatics and 30 healthy subjects) was small,

\begin{tabular}{|c|c|c|c|c|c|c|c|c|c|c|}
\hline \multirow[b]{2}{*}{ Study or Subgroup } & \multicolumn{3}{|c|}{ Intermittent asthmatics } & \multicolumn{3}{|c|}{ Healthy subjects } & \multicolumn{2}{|r|}{ Mean Difference } & \multirow{2}{*}{\multicolumn{2}{|c|}{$\begin{array}{l}\text { Mean Difference } \\
\text { IV, Fixed, } 95 \% \mathrm{Cl}\end{array}$}} \\
\hline & Mean & SD & Total & Mean & SD & Total & Weight & IV, Fixed, $95 \% \mathrm{Cl}$ & & \\
\hline \multicolumn{11}{|l|}{ 9.1.1 Intermittent } \\
\hline Zanconate 2002 & 2.6 & 0.99 & 11 & 2 & 0.92 & 21 & $4.6 \%$ & $0.60[-0.11,1.31]$ & & \\
\hline Uasuf 1999 & 1.39 & 0.65 & 13 & 1.01 & 0.76 & 40 & $12.7 \%$ & $0.38[-0.04,0.80]$ & & - \\
\hline Ohara 2006 & 1.1 & 0.54 & 29 & 1 & 1.37 & 188 & $29.8 \%$ & $0.10[-0.18,0.38]$ & & - \\
\hline Yilmaz 2003 & 1.32 & 0.52 & 47 & 0.94 & 0.5 & 45 & $52.8 \%$ & $0.38[0.17,0.59]$ & & 는 \\
\hline Subtotal $(95 \% \mathrm{Cl})$ & & & 100 & & & 294 & $100.0 \%$ & $0.31[0.16,0.46]$ & & $\bullet$ \\
\hline \multicolumn{11}{|c|}{$\begin{array}{l}\text { Heterogeneity: } \mathrm{Chi}^{2}=3.39, \mathrm{df}=3(\mathrm{P}=0.34) ;\left.\right|^{2}=11 \% \\
\text { Test for owerall effect: } Z=3.97(P<0.0001)\end{array}$} \\
\hline & & & & & & & & & $\begin{array}{cc}-2 & -1 \\
\text { Healthy Subjects }\end{array}$ & $\begin{array}{lcc}0 & 1 & 2 \\
\text { Asthmatic Subjects }\end{array}$ \\
\hline
\end{tabular}

Figure 6 eCO levels in intermittent asthmatics. The WMD of eCO levels between intermittent asthmatics and healthy subjects is $0.31 \mathrm{ppm}(95 \% \mathrm{Cl}$ : 0.16 to 0.46 ) using the fixed effects model. Test for heterogeneity $R=11 \%$. SD: standard deviation; IV: inverse variance; Cl confidence interval; WMD: weighted mean difference; eCO: exhaled carbon monoxide. 


\begin{tabular}{|c|c|c|c|c|c|c|c|c|c|c|}
\hline \multirow[b]{2}{*}{ Stucty or Subgroup } & \multicolumn{3}{|c|}{ Asthmatic Subjects } & \multicolumn{3}{|c|}{ Healthy Subjects } & & \multirow{2}{*}{$\begin{array}{l}\text { Mean Difference } \\
\text { N, Random, 95\% Cl }\end{array}$} & \multirow{2}{*}{\multicolumn{2}{|c|}{$\begin{array}{l}\text { Mean Difference } \\
\text { N, Random, 95\% } \mathrm{Cl}\end{array}$}} \\
\hline & & SD & Total & Mean & SD & Total & Weight & & & \\
\hline \multicolumn{11}{|c|}{ 5.2.1 Mild+Moderate Persistent } \\
\hline Antczak 2005 & 3.1 & 2.04 & 8 & 3.3 & 7.98 & 15 & $0.7 \%$ & $-0.20[-4.48,4.08]$ & & \\
\hline Rosias 2004 & 2.3 & 1.44 & 23 & 2.1 & 2.1 & 9 & $4.7 \%$ & $0.20[-1.29,1.69]$ & & - \\
\hline Montuschi 1999 & 3.45 & 2.02 & 29 & 2.9 & 0.95 & 10 & $9.2 \%$ & $0.55[-0.39,1.49]$ & & - \\
\hline Grover 2008 & 5.76 & 2.24 & 42 & 3.86 & 1.35 & 21 & $9.9 \%$ & $1.90[1.01,2.79]$ & & \\
\hline Yamaya 2001 & 1.55 & 1.04 & 40 & 1.15 & 0.8 & 20 & $17.4 \%$ & $0.40[-0.08,0.88]$ & & - \\
\hline Uasuf 1999 & 2.17 & 0.8 & 16 & 1.01 & 0.76 & 40 & $17.9 \%$ & $1.16[0.70,1.62]$ & & - \\
\hline Ece 2000 & 1.32 & 1.3 & 54 & 0.86 & 1.35 & 235 & $19.4 \%$ & $0.46[0.07,0.85]$ & & $=$ \\
\hline Yilmaz 2003 & 2.05 & 0.9 & 36 & 0.94 & 0.5 & 45 & $20.8 \%$ & $1.11[0.78,1.44]$ & & $=$ \\
\hline Subtotal $(95 \% \mathrm{Cl})$ & & & 248 & & & 395 & $100.0 \%$ & $0.84[0.48,1.20]$ & & 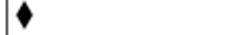 \\
\hline \multicolumn{11}{|c|}{ Heterogeneity: $\mathrm{Tau}^{2}=0.13 ; \mathrm{Chi}^{2}=18.19, \mathrm{df}=7(\mathrm{P}=0.01) ; \mathrm{I}^{2}=62 \%$} \\
\hline \multicolumn{11}{|c|}{ 5.2.2 Severe Persistent } \\
\hline Yamaya 2001 & 4.53 & 5.52 & 31 & 1.15 & 0.8 & 20 & $40.2 \%$ & $3.38[1.41,5.35]$ & & \\
\hline Montuschi 1999 & 4.3 & 1.55 & 15 & 2.9 & 0.95 & 10 & $59.8 \%$ & $1.40[0.42,2.38]$ & & \\
\hline Subtotal $(95 \% \mathrm{Cl})$ & & & 46 & & & 30 & $100.0 \%$ & $2.20[0.29,4.10]$ & & \\
\hline \multicolumn{11}{|c|}{$\begin{array}{l}\text { Heterogeneity: } \operatorname{Tau}^{2}=1.33 ; \mathrm{Chi}^{2}=3.10, \mathrm{df}=1(\mathrm{P}=0.08) ; \mathrm{I}^{2}=68 \% \\
\text { Test for overall effect: } Z=2.26(\mathrm{P}=0.02)\end{array}$} \\
\hline & & & & & & & & & $\begin{array}{cc}-4 & -2 \\
\text { Healthy Subjects }\end{array}$ & $\begin{array}{lcc}0 & 2 & 4 \\
0 & 4 \\
\text { Asthmatic Subjects }\end{array}$ \\
\hline \multicolumn{11}{|c|}{$\begin{array}{l}\text { Figure } 7 \text { eCO levels in mild + moderate persistent and severe persistent asthmatics. The WMD of eCO levels between mild }+ \text { moderate persis- } \\
\text { tent asthmatics and healthy subjects is } 0.84 \mathrm{ppm}(95 \% \mathrm{Cl}: 0.48 \text { to 1.20) using the random effects model. Test for heterogeneity } 12=62 \% \text {. The WMD of } \\
\text { eCO levels between severe persistent asthmatics and healthy subjects is } 2.20 \mathrm{ppm}(95 \% \mathrm{Cl}: 0.29 \text { to } 4.10) \text { using the random effects model. Test for het- } \\
\text { erogeneity } R=68 \% \text {. SD: standard deviation; IV: inverse variance; Cl: confidence interval; WMD: weighted mean difference; eCO: exhaled carbon mon- } \\
\text { oxide. }\end{array}$} \\
\hline
\end{tabular}

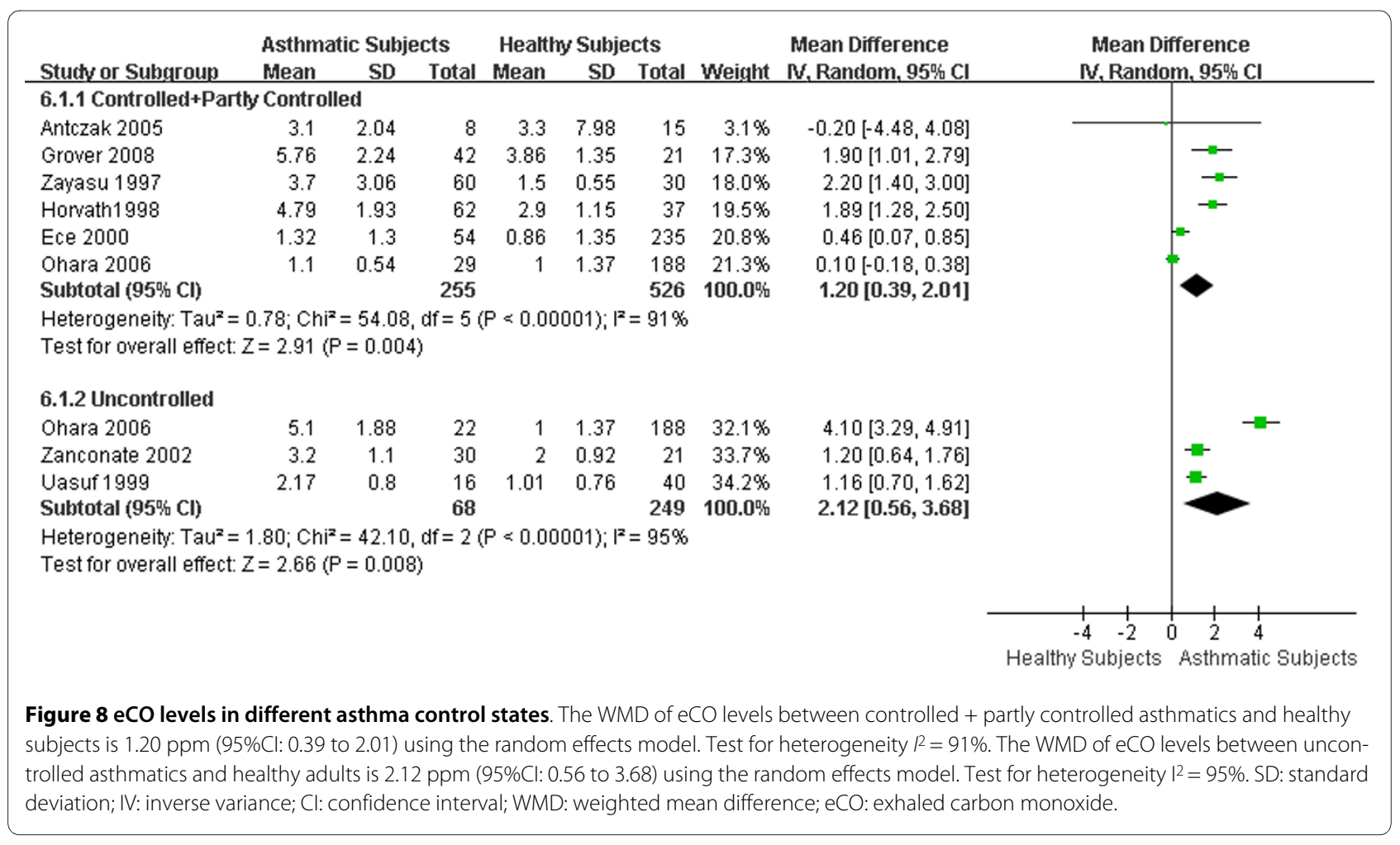




\begin{tabular}{|c|c|c|c|c|c|c|c|c|c|}
\hline \multirow[b]{2}{*}{ Study or Subgroup } & \multicolumn{3}{|c|}{ Before steroid treatment } & \multicolumn{3}{|c|}{ After steroid treatment } & \multirow[b]{2}{*}{ Weight } & \multirow{2}{*}{$\begin{array}{l}\text { Mean Difference } \\
\text { IV, Random, } 95 \% \mathrm{Cl}\end{array}$} & \multirow{2}{*}{$\begin{array}{l}\text { Mean Difference } \\
\text { IV, Random. } 95 \% \mathrm{CI}\end{array}$} \\
\hline & Mean & SD & Total & Mean & SD & Total & & & \\
\hline Yamaya 2001 & 7.6 & 8.9 & 31 & 4.3 & 5.7 & 31 & $7.9 \%$ & $3.30[-0.42,7.02]$ & \\
\hline Zayasu 1997 & 8.4 & 2.08 & 12 & 1.8 & 1.04 & 12 & $14.4 \%$ & $6.60[5.28,7.92]$ & \\
\hline Lim 2000 & 3.28 & 1.1 & 8 & 3.17 & 1.02 & 8 & $15.1 \%$ & $0.11[-0.93,1.15]$ & - \\
\hline Grover 2008 & 5.76 & 2.24 & 42 & 5.21 & 2.24 & 42 & $15.3 \%$ & $0.55[-0.41,1.51]$ & - \\
\hline Yamaya 1999 & 4.6 & 1.79 & 20 & 1.5 & 0.89 & 20 & $15.5 \%$ & $3.10[2.22,3.98]$ & - \\
\hline Khatri 2001 & 3.3 & 0.92 & 22 & 2.6 & 1.3 & 22 & $15.8 \%$ & $0.70[0.03,1.37]$ & - \\
\hline Zanconate 2002 & 3.17 & 1.26 & 30 & 2.7 & 1.07 & 30 & $16.0 \%$ & $0.47[-0.12,1.06]$ & - \\
\hline Total $(95 \% \mathrm{Cl})$ & & & 165 & & & 165 & $100.0 \%$ & $1.98[0.53,3.43]$ & \\
\hline \multicolumn{9}{|c|}{$\begin{array}{l}\text { Heterogeneity: } \text { Tau }^{2}=3.33 ; \mathrm{Chi}^{2}=97.73, \mathrm{df}=6(\mathrm{P}<0.00001) ;\left.\right|^{2}=94 \% \\
\text { Test for overall effect: } Z=2.68(P=0.007)\end{array}$} & $\begin{array}{ccccc}-4 & -2 & 0 & 2 & 4 \\
\text { After steroid treatment } & \text { Before steroid treatment }\end{array}$ \\
\hline
\end{tabular}

Figure 9 eCO levels before and after steroid treatment. The WMD of eCO levels before and after steroid treatment is 1.98 ppm ( $95 \%$ Cl: 0.53 to 3.43) using the random effects model. Test for heterogeneity $R=94 \%$. SD: standard deviation; IV: inverse variance; Cl: confidence interval; WMD: weighted mean difference; eCO: exhaled carbon monoxide.

which decreased the statistical power. A similar reason probably underlies the variability in the uncontrolled asthma group. Thus, further investigation is needed to address whether $\mathrm{eCO}$ could reflect asthma control status.

Our meta-analysis demonstrated that the eCO level in steroid-treated asthmatics was not significantly different from that in steroid-free subjects although the average level of eCO is increased in steroid-free subjects. The lack of statistical significance may relate to the small sample size. In addition, this may possibly also reflect the wide variability in airway inflammation seen in subjects with differing degrees of asthma severity and degrees of control, or the fact that steroids may not affect HO- 1 activity or expression. However, our meta-analysis did demonstrate a significant effect of a formal steroid intervention on eCO levels supporting in vitro data showing that ste-

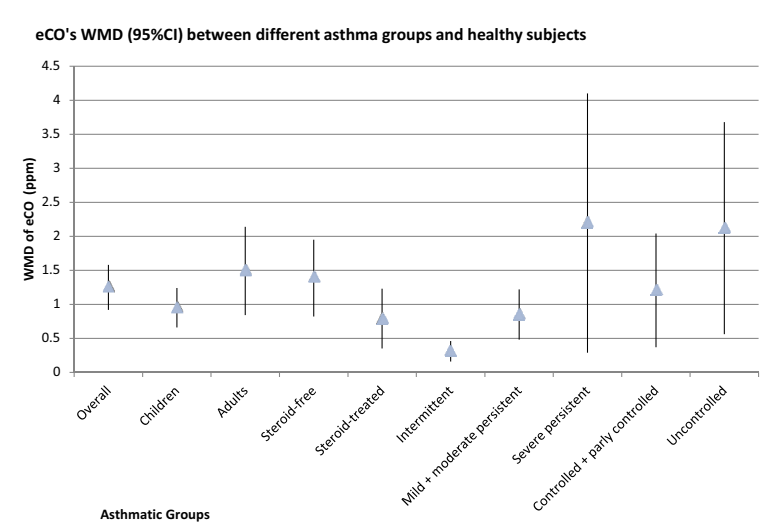

Figure 10 eCO WMD $(95 \% \mathrm{Cl})$ between different asthma groups and healthy subjects. There were no significant differences in eCO levels between steroid-free and steroid-treated asthmatics, mild + moderate persistent and severe persistent asthma, controlled + partly controlled and uncontrolled asthma. However, the eCO level of intermittent asthma was significantly lower than mild + moderate persistent asthma. Cl: confidence interval; WMD: weighted mean difference; eCO: exhaled carbon monoxide; $\boldsymbol{\Lambda}$ : mean of WMD between asthmatics and healthy subjects; vertical line: $95 \% \mathrm{Cl}$ of WMD between asthmatics and subjects. roids are able to directly down-regulate $\mathrm{HO}-1$ promoter activity and expression [39].

Although steroid treatment could reduce the eCO concentrations in asthmatics, the $\mathrm{eCO}$ response might be less sensitive to steroid treatment than that of eNO $[26,33]$. Recent studies demonstrated that using eNO to guide treatment did not result in lower exacerbation frequency as compared to traditional asthma management $[40,41]$. It is possible that the eNO level is too sensitive to steroid treatment, and therefore, the underlying inflammation may still persist despite a significantly decreased eNO level. Hence, treatment based on the less steroid-sensitive eCO level, which correlates with asthma severity to some degree and is reduced after steroid treatment, could be of benefit.

There are some further limitations to using $\mathrm{eCO}$ as an indicator of airway inflammation. The most obvious one is that active and passive smoking status can remarkably affect the eCO level. Both healthy subjects and asthmatic subjects who were current smokers showed significantly higher eCO levels than their nonsmoking control subjects $[42,43]$. The eCO level of some healthy smoking subjects could be much higher than the level of nonsmoking severe persistent asthmatics reported previously [25]. Furthermore, Ece et al [23], demonstrated a significant relationship between passive smoking and $\mathrm{eCO}$ concentration in both healthy and asthmatic children. Thus, when using eCO levels to assess airway inflammation, the active and passive smoking status must be taken into consideration.

Another major limitation is the lack of standardization of eCO measurement. One study included in the metaanalysis used an infrared detection method and the others used an electrochemical method. There was no significant difference in WMD between asthmatics and healthy subjects when the study using infrared method was included (WMD: 1.25 ppm; 95\%CI: 0.92 to 1.58; Random effects model; $I^{2}=75 \%$ ) or not (WMD: $1.33 \mathrm{ppm}$; 95\%CI: 0.99 to 1.66 ; Random effects model; $I^{2}=74 \%$ ). Since the 
eCO values of nonsmoking healthy subjects in this metaanalysis are similar to those reported previously [42] we did not exclude studies due to their different measurements.

\section{Conclusions}

eCO is elevated in non-smoking asthmatics and correlates with disease severity to some extent. Whether $\mathrm{eCO}$ reflects asthma control requires further investigation. Although eCO could not distinguish steroid-treated from steroid-free patients in a cross-sectional analysis, formal steroid treatment significantly reduced eCO levels. eCO might be a potentially useful non-invasive biomarker of airway inflammation and oxidative stress in nonsmoking asthmatics but further work is needed in this area.

\section{List of Abbreviations used}

eCO: exhaled carbon monoxide; AHR: airway hyperresponsiveness; eNO: exhaled nitric oxide; HO: heme oxygenase; ppm: parts per million; SD: standard deviation; CI: confidence interval; SEM: standard error of mean; WMD: weighted mean difference; IV: inverse variance.

\section{Additional material}

Additional file 1 Search Strategy. Document detailing the search strategy used.

Additional file $\mathbf{2}$ Studies included in the meta-analysis examining eCO levels in asthmatics and healthy subjects. Data are expressed as \#mean \pm SEM; * mean \pm SD; $+95 \% C l$; M: male, F: female, $n$ : the number of participants, ppm: parts per million, $\mathrm{FEV}_{1}$ : forced expiratory volume in one second; eCO: exhaled carbon monoxide; ICS: inhaled corticosteroids; L: Iow dose of inhaled corticosteroids; M: medium dose of inhaled corticosteroids; $\mathrm{H}$ : high dose of inhaled corticosteroids.

Additional file 3 Studies included in the meta-analysis examining eCO levels in asthmatics before and after steroid treatment. Data are expressed as \#mean \pm SEM; ${ }^{*}$ mean \pm SD; $+95 \% C l ; M$ : male, F: female, $n$ : the number of participants, ppm: parts per million, $\mathrm{FEV}_{1}$ : forced expiratory volume in one second; eCO: exhaled carbon monoxide; ICS: inhaled corticosteroids; L: low dose of inhaled corticosteroids; M: medium dose of inhaled corticosteroids; $\mathrm{H}$ : high dose of inhaled corticosteroids.

Competing interests

The authors declare that they have no competing interests.

\section{Authors' contributions}

All authors read and met ICMJE criteria for authorship. JZ, MH, XY and RY designed this study. JZ, $X Y$ and $Y S$ extracted data. JZ, $X Y, R Y, J B$ performed the analysis. JZ wrote the first draft of the manuscript. XY, IMA and PJB critically revised. All authors read and approved the final manuscript.

\section{Acknowledgements}

This meta-analysis was supported by National Natural Science Foundation of China (NO.30700342), "the Six Great Talents" (09-B1-001) and Health Promotion Project of Jiangsu Province.

\section{Author Details}

${ }^{1}$ Department of Respiratory Disease, The First Affiliated Hospital of Nanjing Medical University, 300 Guangzhou Road, Nanjing, China, ${ }^{2}$ Department of Epidemiology and Biostatistics, School of Public Health, Nanjing Medical University, 140 Hanzhong Road, Nanjing, China and 3Airway Disease Section, National Heart and Lung Institute, Imperial College, Dovehouse Street, London, UK
Received: 13 January 2010 Accepted: 30 April 2010

Published: 30 April 2010

References

1. Global Initiative for asthma 2008 [http://www.ginasthma.com/]

2. Kharitonov SA, Yates D, Robbins RA, Logan-Sinclair R, Shinebourne EA Barnes PJ: Increased nitric oxide in exhaled air of asthmatic patients. Lancet 1994, 343(8890):133-135.

3. Persson MG, Zetterstrom O, Agrenius V, Ihre E, Gustafsson LE: Singlebreath nitric oxide measurements in asthmatic patients and smokers. Lancet 1994, 343(8890):146-147

4. Habib SS: Elevated exhaled nitric oxide (NO) in asymptomatic asthmatics taking bronchodilators on demand with controlled body composition. J Pak Med Assoc 2009, 59(3):147-150.

5. Kharitonov SA, Yates DH, Barnes PJ: Inhaled glucocorticoids decrease nitric oxide in exhaled air of asthmatic patients. Am J Respir Crit Care Med 1996, 153(1):454-457.

6. Baraldi E, Azzolin NM, Zanconato S, Dario C, Zacchello F: Corticosteroids decrease exhaled nitric oxide in children with acute asthma. J Pediatr 1997, 131(3):381-385.

7. Jarvis MJ, Russell MA, Saloojee Y: Expired air carbon monoxide: a simple breath test of tobacco smoke intake. BrMed J 1980, 281(6238):484-485.

8. Zayasu K, Sekizawa K, Okinaga S, Yamaya M, Ohrui T, Sasaki H: Increased carbon monoxide in exhaled air of asthmatic patients. Am J Respir Crit Care Med 1997, 156(4 Pt 1):1140-1143.

9. Horvath I, Donnelly LE, Kiss A, Paredi P, Kharitonov SA, Barnes PJ: Raised levels of exhaled carbon monoxide are associated with an increased expression of heme oxygenase- 1 in airway macrophages in asthma: a new marker of oxidative stress. Thorax 1998, 53(8):668-672.

10. Kharitonov SA, Barnes PJ: Exhaled markers of pulmonary disease. American journal of respiratory and critical care medicine 2001, 163(7):1693-1722

11. Zetterquist W, Marteus H, Johannesson M, Nordvall SL, Ihre E, Lundberg JON, Alving K: Exhaled carbon monoxide is not elevated in patients with asthma or cystic fibrosis. European Respiratory Journal 2002 20(1):92-99.

12. Kong PM, Chan CC, Lee P, Wang YT: An assessment of the role of exhaled carbon monoxide in acute asthmatic exacerbations in hospitalised patients. Singapore Med J 2002,43(8):399-402.

13. Paredi P, Biernacki W, Invernizzi G, Kharitonov SA, Barnes PJ: Exhaled carbon monoxide levels elevated in diabetes and correlated with glucose concentration in blood: a new test for monitoring the disease? Chest 1999, 116(4):1007-1011.

14. Zegdi R, Perrin D, Burdin M, Boiteau R, Tenaillon A: Increased endogenous carbon monoxide production in severe sepsis. Intensive Care Med 2002, 28(6):793-796

15. Begg CB, Mazumdar M: Operating characteristics of a rank correlation test for publication bias. Biometrics 1994, 50(4):1088-1101.

16. Egger M, Davey Smith G, Schneider M, Minder C: Bias in meta-analysis detected by a simple, graphical test. BMJ 1997, 315(7109):629-634.

17. Antczak A, Kharitonov SA, Montuschi P, Gorski P, Barnes PJ: Inflammatory response to sputum induction measured by exhaled markers. Respiration 2005, 72(6):594-599.

18. Zayasu J, Sekizawa K, Okinaga S, Yamaya M, Ohrui A, Sasaki H: Increased carbon monoxide in exhaled air of asthmatic patients. American Journal of Respiratory and Critical Care Medicine 1997, 156(4 I):1140-1143.

19. Horvath I, Donnelly LE, Kiss A, Paredi P, Kharitonov SA, Barnes PJ: Raised levels of exhaled carbon monoxide are associated with an increased expression of heme oxygenase- 1 in airway macrophages in asthma: $A$ new marker of oxidative stress. Thorax 1998, 53(8):668-672.

20. Montuschi P, Corradi M, Ciabattoni G, Nightingale J, Kharitonov SA, Barnes $P J$ : Increased 8-isoprostane, a marker of oxidative stress, in exhaled condensate of asthma patients. American Journal of Respiratory and Critical Care Medicine 1999, 160(1):216-220.

21. Paredi P, Leckie MJ, Horvath I, Allegra L, Kharitonov SA, Barnes PJ: Changes in exhaled carbon monoxide and nitric oxide levels following allergen challenge in patients with asthma. European Respiratory Journal 1999, 13(1):48-52

22. Uasuf CG, Jatakanon A, James A, Kharitanov SA, Wilson NM, Barnes PJ: Exhaled carbon monoxide in childhood asthma. Journal of Pediatrics 1999, 135(5):569-574 
23. Ece A, Gurkan F, Haspolat K, Derman O, Kirbas G: Passive smoking and expired carbon monoxide concentrations in healthy and asthmatic children. Allergologia et Immunopathologia 2000, 28(5):255-260.

24. Khatri SB, Ozkan M, McCarthy K, Laskowski D, Hammel J, Dweik RA, Erzurum SC: Alterations in exhaled gas profile during allergen-induced asthmatic response. American Journal of Respiratory and Critical Care Medicine 2001, 164(10 I):1844-1848.

25. Yamaya M, Hosoda M, Ishizuka S, Monma M, Matsui T, Suzuki T, Sekizawa $\mathrm{K}$, Sasaki H: Relation between exhaled carbon monoxide levels and clinical severity of asthma. Clinical and Experimental Allergy 2001 31(3):417-422

26. Zanconato S, Scollo M, Zaramella C, Landi L, Zacchello F, Baraldi E: Exhaled carbon monoxide levels after a course of oral prednisone in children with asthma exacerbation. Journal of Allergy and Clinical Immunology 2002, 109(3):440-445.

27. Yilmaz O, Ozturk F, Bakirtas A, Cengizlier R, Turktas I: Exhaled carbon monoxide levels in children with bronchial asthma. Pediatric Asthma, Allergy and Immunology 2003, 16(3):155-162.

28. Beck-Ripp J, Latzin P, Griese M: Exhaled carbon monoxide is not flow dependent in children with cystic fibrosis and asthma. European journal of medical research 2004, 9(11):518-522.

29. Rosias PPR, Dompeling E, Dentener MA, Pennings HJ, Hendriks HJE, Van lersel MPA, Jobsis Q: Childhood asthma: Exhaled markers of airway inflammation, asthma control score, and lung function tests. Pediatric Pulmonology 2004, 38(2):107-114.

30. Ohara Y, Ohrui T, Morikawa T, He M, Yasuda H, Yamaya M, Sasaki H: Exhaled carbon monoxide levels in school-age children with episodic asthma. Pediatric Pulmonology 2006, 41(5):470-474.

31. Grover RS, Kumar R: Exhaled carbon monoxide levels: As a marker of clinical severity and control of asthma. Journal of Asthma 2008, 45(8):677-680

32. Lim S, Groneberg D, Fischer A, Oates T, Caramori G, Mattos W, Adcock I, Barnes PJ, Chung KF: Expression of heme oxygenase isoenzymes 1 and 2 in normal and asthmatic airways: Effect of inhaled corticosteroids. American Journal of Respiratory and Critical Care Medicine 2000, 162(5):1912-1918.

33. Kharitonov SA, Donnelly LE, Montuschi P, Corradi M, Collins JV, Barnes PJ: Dose-dependent onset and cessation of action of inhaled budesonide on exhaled nitric oxide and symptoms in mild asthma. Thorax 2002 57(10):889-896.

34. Yamaya M, Sekizawa K, Ishizuka S, Monma M, Sasaki H: Exhaled carbon monoxide levels during treatment of acute asthma. European Respiratory Journal 1999, 13(4):757-760.

35. Horvath I, Barnes PJ: Exhaled monoxides in asymptomatic atopic subjects. Clin Exp Allergy 1999, 29(9):1276-1280.

36. Paredi P, Leckie MJ, Horvath I, Allegra L, Kharitonov SA, Barnes PJ: Changes in exhaled carbon monoxide and nitric oxide levels following allergen challenge in patients with asthma. European Respiratory Journal 1999, 13(1):48-52.

37. Lim S, Groneberg D, Fischer A, Oates T, Caramori G, Mattos W, Adcock I, Barnes PJ, Chung KF: Expression of heme oxygenase isoenzymes 1 and 2 in normal and asthmatic airways: effect of inhaled corticosteroids. Am J Respir Crit Care Med 2000, 162(5):1912-1918.

38. Harju T, Soini Y, Paakko R, Kinnula VL: Up-regulation of heme oxygenaseI in alveolar macrophages of newly diagnosed asthmatics. Respir Med 2002, 96(6):418-423.

39. Lavrovsky Y, Drummond GS, Abraham NG: Downregulation of the human heme oxygenase gene by glucocorticoids and identification of $56 \mathrm{~b}$ regulatory elements. Biochem Biophys Res Commun 1996, 218(3):759-765.

40. Smith AD, Cowan JO, Brassett KP, Herbison GP, Taylor DR: Use of exhaled nitric oxide measurements to guide treatment in chronic asthma. $N$ Engl J Med 2005, 352(21):2163-2173.

41. Shaw DE, Berry MA, Thomas M, Green RH, Brightling CE, Wardlaw AJ, Pavord ID: The use of exhaled nitric oxide to guide asthma management: a randomized controlled trial. Am J Respir Crit Care Med 2007, 176(3):231-237.

42. Middleton ET, Morice $\mathrm{AH}$ : Breath carbon monoxide as an indication of smoking habit. Chest 2000, 117(3):758-763.

43. Sato S, Nishimura K, Koyama H, Tsukino M, Oga T, Hajiro T, Mishima M: Optimal Cutoff Level of Breath Carbon Monoxide for Assessing
Smoking Status in Patients with Asthma and COPD. Chest 2003 124(5):1749-1754.

doi: 10.1186/1465-9921-11-50

Cite this article as: Zhang et al., Exhaled carbon monoxide in asthmatics: a meta-analysis Respiratory Research 2010, 11:50

\section{Submit your next manuscript to BioMed Central and take full advantage of:}

- Convenient online submission

- Thorough peer review

- No space constraints or color figure charges

- Immediate publication on acceptance

- Inclusion in PubMed, CAS, Scopus and Google Scholar

- Research which is freely available for redistribution

Submit your manuscript at www.biomedcentral.com/submit
C Biomed Central 